\title{
Smear positive pulmonary tuberculosis among HIV patients receiving Highly Active Antiretroviral Therapy in Dar es Salaam, Tanzania
}

\author{
VENERANDA BWANA ${ }^{1 *}$, FILEMONI TENU ${ }^{1}$, STEPHEN M. MAGESA ${ }^{1}$ and ${ }^{2}$ SAYOKI G. \\ MFINANGA \\ ${ }^{1}$ National Institute for Medical Research, Amani Research Centre, P.O. Box 81, Muheza, Tanzania \\ ${ }^{2}$ National Institute for Medical Research, Muhimbili Research Centre, Dar es Salaam, Tanzania
}

\begin{abstract}
Globally, tuberculosis-HIV co-infections are on the increase. In 2007, 15\% (1.37 million) of the tuberculosis cases were HIV-positive tuberculosis (TB). This cross-sectional study was conducted in February 2009 to assess the effect of the level of CD4 lymphocyte counts on the development of smear positive pulmonary TB (PTB) among HIV patients before and after initiation of highly active antiretroviral therapy (HAART). A total of $155 \mathrm{HIV}$ patients who were on HAART programme were enrolled and out of these $42(27.1 \%)$ were smear positive PTB. Of the 42 PTB patients, $38(90.5 \%)$ were also infected with HIV and were already at initiation of HAART. There was no association between the development of smear positive PTB and socio-demographic characteristics among HIV patients before and after HAART initiation $(P>0.05)$. A larger proportion of HIV+PTB patients diagnosed before and after HAART initiation was found with CD4 lymphocyte count $<200 \mathrm{cells} / \mu \mathrm{l}$. However, the difference was not statistically significant $(P=0.092)$. Among HIV patients who were diagnosed to be smear positive PTB after HAART initiation, their CD4 lymphocyte counts at time of TB diagnosis was lower than their CD4 lymphocyte counts at time of HAART initiation. The four patients diagnosed with PTB after HAART initiation had mean CD4 lymphocyte counts at HAART initiation not statistically different from that at TB diagnosis ( $\mathrm{t}=0.715$, $P=0.526)$. The median time period within which the diagnosis of smear positive PTB was made after HAART initiation was 22 weeks and the mean time was 66.75 weeks. These findings provide evidence that development of smear positive PTB after HAART initiation may occur at any level of CD4 lymphocyte count $(P<0.05)$. This study was limited by the relatively small sample size, we therefore recommend more studies involving a larger sample size in order to estimate more accurately the effect of both level of CD4 lymphocyte count and HAART on the development of smear positive PTB among HIV patients on treatment.
\end{abstract}

Key words: Pulmonary tuberculosis, HIV, HAART, CD4 lymphocyte, Tanzania

\section{Introduction}

Tuberculosis (TB) is one of the major public health problems globally. An estimated onethird of the world's population is infected with the Mycobacterium tuberculosis, and the disease kills nearly 1.7 million people each year (WHO, 2009). On the other hand, HIV is currently, the most important predisposing factor for developing TB in individuals coinfected with M. tuberculosis. Tuberculosis-HIV co-infection is increasing at an alarming rate. In 2006 there were 9.2 million incident tuberculosis cases in the world, and among these 0.7 million cases were HIV-positive. In 2007, there were at least 1.37 million cases of HIV-positive TB i.e. about 15\% of the total incidence (WHO, 2009). In Africa, TB is often the first manifestation of HIV infection, and it is the leading cause of death among HIVinfected patients. Furthermore in parts of southern Africa more than $60 \%$ of TB patients are HIV positive (Badri et al., 2002; Corbett et al., 2003; WHO, 2008; Williams \& Dye, 2003).

\footnotetext{
* Correspondence: Dr. Veneranda Bwana; E-mail: vbwana@nimr.or.tz
} 
The reported number of TB cases in Tanzania has increased six-fold between 1983 and 2006, and the majority of the cases occurred in young adults (15-44 years), the same age group which is mostly affected by HIV/AIDS (MoHSW, 2006). In Tanzania, the HIV prevalence among new smear-positive TB patients increased from 28\% in 1991-1993 to 40\% in 1994-1998 (Range et al., 2001). The distribution of TB in the country varies from region to region. For instance, in Dar es Salaam Region with 6\% of the population contributes about $24 \%$ of all cases of TB in Tanzania (MoHSW, 2006). Besides that, Kwesigabo et al. (1999) showed that the prevalence of pulmonary TB among HIV-1 patients was $59 \%$ among hospitalized patients in Kagera Region in north-western Tanzania.

Tuberculosis case-fatality rate in Africa is 16-35\% in HIV-positive cases not receiving antiretroviral treatment and $4-9 \%$ in HIV-negative cases (van den Broek et al., 1998; Mukadi et al., 2001). Increased mortality during the first month of treatment seems largely attributable to the TB itself (Mukadi et al., 2001). Studies in Tanzania have indicated that a proportion of patients on ART develop TB after starting ART, with about $50 \%$ during the first three months of treatment (Somi et al., 2009). Moreover, studies have shown showed high incidence rates of PTB within the first three months after initiation of highly active antiretroviral therapy (HAART) initiation for patients with notified TB in Cambodia (76.3\%), Thailand (52.5\%), Kenya (51.9\%), Malawi (66.7\%) and Cameroon $(80.0 \%)$ (Bonnet et al., 2006). The greatest proportion of HIV positive associated TB patients are found at CD4 lymphocyte count $<200$ cells/ $\mu l$, and this is associated with highest mortality rates (Ackah et al., 1995). However several studies from different countries have showed that antiretroviral drugs reduce the incidence of TB in HIV-infected people by $80 \%$ or more, with the greatest effect at the lowest CD4 lymphocyte counts (Girardi et al., 2000; Badri et al., 2002).

Tuberculosis is an aggressive opportunistic infection that arises at higher median CD4 lymphocyte count compared with other AIDS-related diseases conditions. In Cote d'Ivoire, smear-positive TB patients presented with a median CD4 lymphocyte count of 257 cells/ $\mu$ l (Ackah et al., 1995). However, the World Health Organization current guidelines recommend treatment for all HIV patients at a higher CD4 lymphocyte count threshold of 350 cells/ $\mu \mathrm{l}$ regardless of symptoms of HIV (WHO, 2009). Therefore, the potential effect of antiretroviral treatment on the TB incidence is reduced because many HIV-infected patients with TB present before antiretroviral drugs are prescribed. In contrary, Lawn et al. (2005) showed that TB may develop even among individuals who are responding to HAART. This suggests that TB incidence and mortality will be reduced if antiretroviral drug coverage is high, start early and is combined with TB preventive treatment (Williams \& Dye., 2003).

Tuberculosis prevention and control in countries with high burden of TB disease like Sub-Saharan Africa aims mainly at case detection and treatment through a well adopted (Directly Observed Therapy, Short course (DOTS) programme (Harries et al., 2002). Tuberculosis control and prevention has been a major challenge since the the beginning of HIV pandemic and these two diseases continue to call attention to most scientists and reseachers in order to develop interventions strategies to pave the way on how to combat these two killer diseases. Therefore there is a need to allocate more sustainable preventive strategies especially in poor resource and developing countries. The objective of this study was therefore, to assess the effect of the level of CD4 lymphocyte 
count on the development of smear positive pulmonary TB among HIV patients before and after HAART initiation. Specifically, the study aimed (i) to estimate the time on which the diagnosis of smear positive PTB was made; (ii) to estimate the effect of smear positive pulmonary TB on the level of CD4 lymphocyte count at initiation of HAART; and (iii) to estimate the effect of HAART on the development of smear positive pulmonary TB

\section{Materials and Methods}

\section{Study area and population}

This cross sectional study of HIV-positive patients on HAART was carried out at Amana Hospital HIV Clinic in Dar es Salaam, Tanzania during February 2009. The study population were non-pregnant HIV positive patients on HAART, aged 15-49 years old living in Dar es Salaam. Children and pregnant women were not included as they follow a special HIV programme. Patients with chronic conditions like diabetes, chronic renal failure, cancer and malnutrition were excluded. Patients with smear negative pulmonary TB and extra pulmonary TB were also excluded. Patients with immune reconstitution syndrome and drug failure were noted with care. The study site, Amana HIV Clinic, was randomly selected among all other clinics in Dar es salaam City. Convenient sampling method was the main method that was used during selection of study subjects in every section at Care and Treatment Centre (CTC) and TB/HAART. All eligible study subjects who attended the clinic during February 2009 and agreed to participate in the study were included.

The sample size of the study was estimated to be 125 HIV associated PTB patients where by n1=36, (number of PTB patients after HAART initiation) and n2=87, (number of PTB patients before HAART initiation. Sample size calculated using Stata Version 10 (Statacorp, 4905 Lakeway Drive, Texas, USA).

\section{Data collection}

A questionnaire was used for data collection and it was structured based on knowledge background of the HIV related infection as well as all co-morbidity associated with HIV infection. It was composed on basic socio-demographic characteristics, prior history of TB, behaviour on ART adherence and smoking. Furthermore other clinical diagnostic information of co-morbidity including TB, clinical stage of HIV, treatment of the patient, CD4 lymphocyte counts and sputum smear for acid fast bacilli was extracted from the patient's case file notes.

Information was collected at exit interviews and from the clinical records by using a pre-tested questionnaire. All patients were two months old and above on HAART at the time of data collection. At each section (CTC and TB/HAART) all patients who had started HAART and were conveniently enrolled were identified.

\section{Data analysis}

All questionnaires were assigned a serial number. The data collected was double entered, cleaned and coded into an database developed using Epi Info version 3.512008 (Centers for Disease Control and Prevention, Atlanta, GA,USA). Data analysis was done using SPSS version 16 for windows (SPSS Inc, Chicago, IL, USA). The analysis involved comparison within the individual group characteristics and across both groups (Tuberculous and non 
tuberculous patients). Pearson Chi-square, Fisher's exact test were used to compare group difference between categorical variables. Paired sample t- test was used to compare the observed mean differences of CD4 lymphocyte counts at HAART initiation and at TB diagnosis after HAART initiation.

\section{Ethical consideration}

Ethical clearance was obtained from Medical Research Coordinating Committee of the Tanzania National Institute for Medical Research. Written informed consent was obtained from study participants before enrolment. Confidentiality was strictly maintained for study subjects.

\section{Results}

A total of $155 \mathrm{HIV}$ patients who were on HAART programme were enrolled and out of these $42(27.1 \%)$ were smear positive pulmonary TB (PTB). Of the smear positive PTB, 38 (90.5\%) were already at initiation of HAART. Of the 42 PTB patients, 38 (90.5\%) were also infected with HIV. Most (36/42) of the patients were in the age group 30-39 years and the majority were on formal employment (31/42). Slightly over one-third (35.7\%) of the TB-HIV patients were married. The rest were single, widowed or separated (Table 1).

Table 1: Socio-demographic characteristics among HIV patients before and after HAART initiation

\begin{tabular}{|c|c|c|c|}
\hline \multirow{3}{*}{$\begin{array}{l}\text { Social demographic } \\
\text { characteristics }\end{array}$} & \multicolumn{2}{|c|}{ Smear positive PTB } & \multirow{2}{*}{$\begin{array}{l}\text { Non Tuberculous } \\
\text { Patients }\end{array}$} \\
\hline & Before HAART & After HAART & \\
\hline & $\mathrm{N}(\%)$ & $\mathrm{N}(\%)$ & $\mathrm{N}(\%)$ \\
\hline \multicolumn{4}{|l|}{ Age(years) } \\
\hline $15-29$ & $6(16 \%)$ & $0(0 \%)$ & $16(14 \%)$ \\
\hline $30-49$ & $32(84 \%)$ & $4(100 \%)$ & $97(86 \%)$ \\
\hline \multicolumn{4}{|l|}{ Sex } \\
\hline Males & $18(47 \%)$ & $1(25 \%)$ & $42(37 \%)$ \\
\hline Females & $20(53 \%)$ & $3(75 \%)$ & $71(63 \%)$ \\
\hline \multicolumn{4}{|l|}{ HAART line treatment } \\
\hline $1^{\text {st line }}$ & $38(100 \%)$ & $4(100 \%)$ & $110(97 \%)$ \\
\hline $2^{\text {nd }}$ line & $0(0 \%)$ & $0(0 \%)$ & $3(3 \%)$ \\
\hline \multicolumn{4}{|l|}{ Occupation } \\
\hline Formal employment & $27(71 \%)$ & $4(100 \%)$ & $95(84 \%)$ \\
\hline Informal employment & $11(29 \%)$ & $0(0 \%)$ & $18(16 \%)$ \\
\hline \multicolumn{4}{|l|}{ Education } \\
\hline Primary & $28(74 \%)$ & $0(0 \%)$ & $70(62 \%)$ \\
\hline Secondary and above & $10(26 \%)$ & $4(100 \%)$ & $43(38 \%)$ \\
\hline \multicolumn{4}{|l|}{ Smoking status } \\
\hline Current and previous & $11(29 \%)$ & $0(0 \%)$ & $24(21 \%)$ \\
\hline Never smoke & $27(71 \%)$ & $4(100 \%)$ & $89(79 \%)$ \\
\hline \multicolumn{4}{|l|}{ Marital status } \\
\hline Single/Separate/widow & $24(63 \%)$ & $3(75 \%)$ & $58(51 \%)$ \\
\hline Married & $14(37 \%)$ & $1(25 \%)$ & $55(49 \%)$ \\
\hline Total & 38 & 4 & 113 \\
\hline
\end{tabular}


All HIV associated pulmonary TB patients were in first line of HAART treatment (three drug combinations; Zidovudine (AZT) + Lamivudine (3TC) + Nevirapine (NVP) or Zidovudine (AZT) + Lamivudine (3TC) + Efavirenz (EFV) or Stavudine $(\mathrm{d} 4 \mathrm{~T})+$ Lamivudine $(3 \mathrm{TC})+$ Nevirapine $(\mathrm{NVP})$ or Stavudine $(\mathrm{d} 4 \mathrm{~T})$ + Lamivudine $(3 \mathrm{TC})+$ Efavirenz $(\mathrm{EFV})$. There was no association between the development of smear positive PTB and sociodemographic characteristics among HIV patients before and after HAART initiation $(P>0.05)$ (Table 1).

Table 2: Proportion distribution among HIV patients according to levels of CD4 lymphocyte count before and after HAART

\begin{tabular}{|c|c|c|c|}
\hline \multirow{3}{*}{  } & \multicolumn{2}{|c|}{ Smear positive PTB } & \multirow{2}{*}{$\begin{array}{l}\text { Non Tuberculous } \\
\text { Patients }\end{array}$} \\
\hline & Before HAART & After HAART & \\
\hline & N (\%) & $\mathbf{N}(\%)$ & N (\%) \\
\hline \multicolumn{4}{|l|}{ At HIV diagnosis* } \\
\hline $1-200$ & $29(78 \%)$ & $2(50 \%)$ & $81(82 \%)$ \\
\hline $201-500$ & $8(22 \%)$ & $2(50 \%)$ & $18(18 \%)$ \\
\hline Total & 37 & 4 & 99 \\
\hline \multicolumn{4}{|l|}{ At TB diagnosis di* $^{*}$} \\
\hline $1-200$ & $29(78 \%)$ & $4(100 \%)$ & \\
\hline $201-500$ & $8(22 \%)$ & $0(0 \%)$ & \\
\hline Total & 37 & 4 & \\
\hline \multicolumn{4}{|l|}{ At HAART initiation ${ }^{* * *}$} \\
\hline $1-200$ & & $2(50 \%)$ & $86(86 \%)$ \\
\hline $201-500$ & & $2(50 \%)$ & $14(14 \%)$ \\
\hline Total & & 4 & 100 \\
\hline
\end{tabular}

A larger proportion of HIV+PTB patients diagnosed before and after HAART initiation was found with CD4 lymphocyte count less than 200cells/ $\mu$ l. However, the difference was not statistically significant $(P=0.092)$ (Table 2).

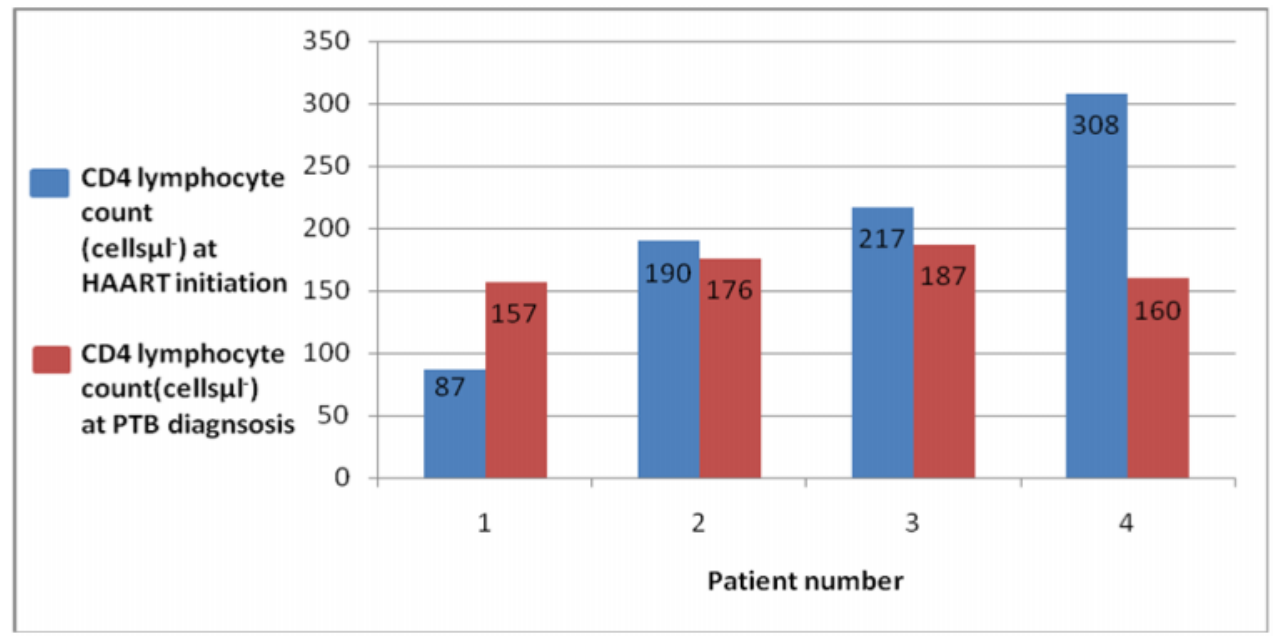

Figure 1: Level of CD4 lymphocyte counts at smear positive PTB diagnosis after HAART initiation compared to that at HAART initiation 
The CD4 lymphocyte count among HIV patients who were diagnosed to be smear positive PTB after HAART initiation was lower than at time of HAART initiation (Figure 1). The four patients diagnosed with PTB after HAART initiation had their mean CD4 lymphocyte counts at HAART initiation not statistically different from that at TB diagnosis $(\mathrm{t}=0.715$, $P=0.526)$. Their median CD4 lymphocyte counts at time of TB diagnosis was 168 cells/ $\mu 1$ (range $=157-187 \mathrm{cells} / \mu \mathrm{l}$ ) lower than at HAART initiation $(204 \mathrm{cells} / \mu \mathrm{l}$; range $=87-308$ cells/ $\mu \mathrm{l})$.

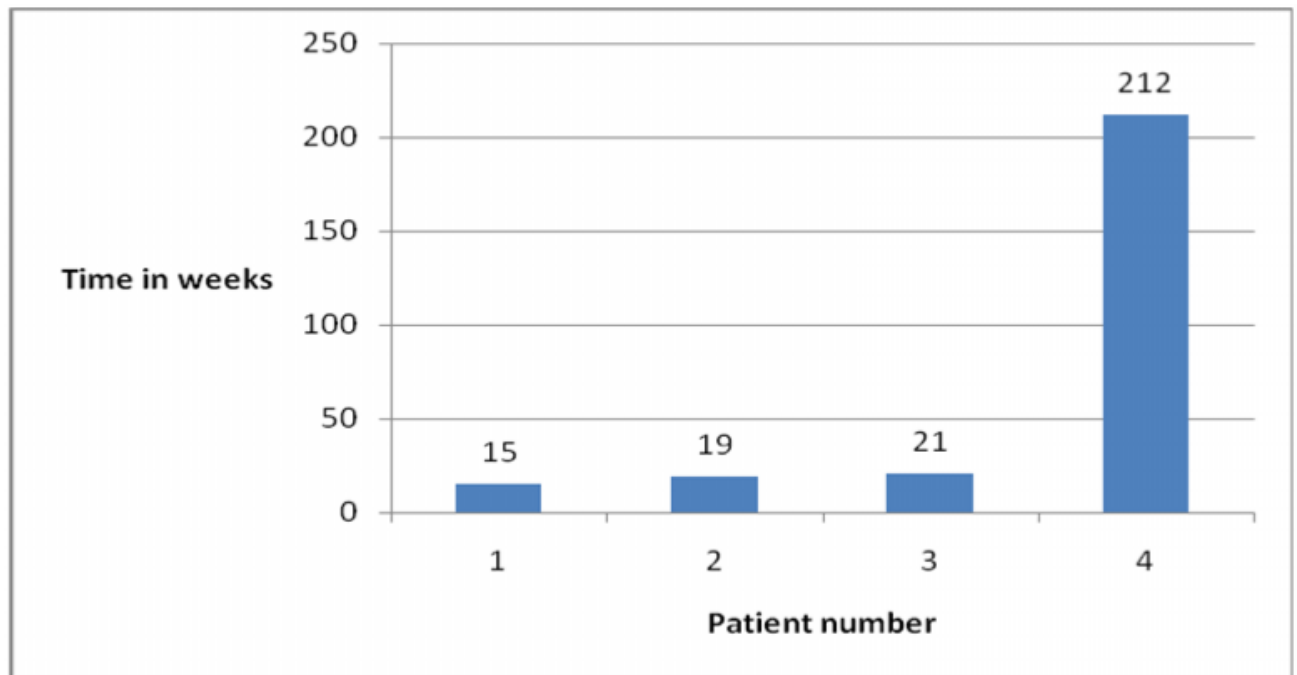

Figure 2: Time in weeks at which patients diagnosed to be smear positive PTB since initiated HAART

The median time period within which the diagnosis of smear positive PTB was made after HAART initiation was 22 weeks and the mean time was 66.75 weeks (Range 15-212 weeks) (Figure 2).

\section{Discussion}

Tuberculosis remains a major public health problem and a burden among people living with HIV infection. In this study, over $90 \%$ of the TB patients were also infected with HIV. In developing countries TB is the most common life-threatening opportunistic infection in patients with HIV/AIDS (van den Broek et al., 1998; Range et al., 2001; Corbett et al., 2003; Sharma et al., 2005; Somi et al., 2009). By the end of 2000, about 11.5 million people were coinfected with HIV and M. tuberculosis, globally; 70\% of co-infected people were in subSaharan Africa (Sharma et al., 2005). However many HIV patients show up late at health facility and presented with low levels of CD4 lymphocyte counts this means that the disease progression has already.

In this study all smear positive pulmonary $\mathrm{TB}$ patients were on the first line of HAART and they had no history of chronic debilitated conditions. The results showed that of the four studied patients who were diagnosed with PTB after HAART initiation their observed mean differences of CD4 lymphocyte counts at HAART initiation and at TB diagnosis were not statistically different. These data provide evidence that smear positive PTB arise after HAART initiation at any CD4 lymphocyte counts. However, WHO current 
guidelines recommend treatment for all HIV patients at a higher CD4 lymphocyte count threshold of 350 cells/ $\mu$ l regardless of symptoms of HIV (WHO, 2009). It has been observed that, HAART, through immune restoration, reduces the TB incidence among people living with HIV infection (Girardi et al., 2000; Badri et al., 2002; Bonnet et al., 2006). The finding of this study have great implication on starting of HAART according to the threshold of CD4 cell count, although the findings observed from a small number of studied patients.

Studies done elsewhere have demonstrated that antiretroviral drugs reduce the incidence of TB in HIV-infected patients by $80 \%$ or more (Girardi et al., 2000; Badri et al., 2002). In contrary the findings of this study explain the effects of HAART on the development of smear positive pulmonary TB but since the sample size is small it remain and justify a further study to fully provide this evidence.

The results showed that of the four studied patients who were diagnosed PTB after HAART initiation their median duration of weeks between TB diagnosis and at HAART initiation was found to be 22 weeks. These findings suggest that these patients had likely to have undiagnosed prevalent TB at HAART initiation, or subclinical TB which becomes apparent during the immune reconstitution syndrome. However, if this syndrome occurs, it is more likely within the first three months after HAART initiation. But due to the limited availability of diagnostic tools would make it impossible to distinguish between immune reconstitution inflammatory syndrome and undiagnosed prevalent tuberculosis which is a common problem in resource poor settings (Bonnet et al., 2006). Despite responding well to antiretroviral treatment, the four patients developed PTB after starting HAART. However, this hypothesis will need to be proved in a study with adequate sample size of patients diagnosed with PTB. Thus, HIV disease still remain to be the major triggerring factor for the development of PTB among HIV infected patients.

This study had some limitations. The small sample size of patients diagnosed with PTB could not allow adequate statistical tests for comparative analysis. Moreover, there was selection bias because patients were conveniently selected at TB/HAART and CTC sections after finishing their daily routine follow up. These patients might differ from those the general population. Moreover, a total of 28 patients refused to participate in the study. These patients could have different characteristics compared to those who participated in the study.

In conclusion, more studies should be done in order to answer the hypothesis developed from our study findings on the effect of both level of CD4 lymphocyte count and HAART on the development of smear positive PTB among patients on HAART. A cohort study of HIV patients before and after initiation of HAART is highly recommended.

\section{Acknowledgements}

The authors acknowledge the support of Dr. Guerino Chalamila and all health staffs of Amana Care and Treatment Clinic in the recruitment of study participants. Many thanks to all patients who agreed to participate in this study.

\section{References}

Ackah, A.N., Coulibaly, D., Digbeu, H., Diallo, K., Vetter, K.M., Coulibaly, I.M., Greenberg, A.E. \& Decock, K.M. (1995) Response to treatment, mortality, and CD4 lymphocyte 
counts in HIV-infected persons with tuberculosis in Abidjan, Cote d'Ivoire. Lancet 345, 607-610.

Badri, M., Wilson, D. \& Wood, R. (2002) Effect of highly active antiretroviral therapy on incidence of tuberculosis in South Africa: a cohort study. Lancet 359, 2059-2064.

Bonnet, M., Pinoges, L., Varaine, F., Oberhauser, B., O’Brien, D., Kebede, Y., Hewison, C., Zacharia, R. \& Ferradini, L.(2006) Tuberculosis after HAART initiation in HIVpositive patients from five countries with a high tuberculosis burden. AIDS 20, 1275-1279.

Corbett, E.L., Watt, C.J., Walker, N., Maher, D., Williams, B.G., Raviglione, M.C. \& Dye, C. (2003) The growing burden of tuberculosis global trends and interactions with the HIV epidemic. Archives of Internal Medicine 63, 1009-1021.

Giradi, E., Antonucci, G., Vanacore, P., Libanore, M., Errante, I., Matteelli, A. \& Ippolito, G. (2000) Impact of combination antiretroviral therapy on the risk of tuberculosis among persons with HIV infection. AIDS 14, 1985-1991.

Harries, A.D., Hargreaves, N.J., Chimzizi, R., Salaniponi, F.M. (2002) Highly active antiretroviral therapy and tuberculosis control in Africa: synergies and potential. Bulletin of the World Health Organization 80, 464-469.

Kwesigabo, G., Killewo, J.Z.J., Sandström, A.M., Winani, S., Mhalu, F.S., Biberfeld, G. \& Wall, S. (1999) Prevalence of HIV infection among hospital patients in North West Tanzania. AIDS Care 11, 87-93.

Lawn, D.S., Badri, M. \& Wood, R. (2005) Tuberculosis among HIV-infected patients receiving HAART: long term incidence and risk factors in a South African cohort. AIDS 9, 2109-2116.

MoHSW (2006) National Tuberculosis and Leprosy Programme. Annual report 2006. Ministry of Health and Social Welfare, Dar es Salaam, Tanzania.

Mukadi, Y.D., Maher, D. \& Harries, A. (2001) Tuberculosis case fatality rates in high HIV prevalence populations in sub-Saharan Africa. AIDS15, 143-52.

Range, N., Ipuge, Y.A., O’Brien, R.J., Egwaga, S.M., Mfinanga, S.G., Chonde, T.M., Mukadi, Y.D. \& Borgdorff, W.M. (2001) Trend in HIV prevalence among tuberculosis patients in Tanzania, 1991-1998. International Journal of Tuberculosis Lung Disease 5, 405-412.

Sharma, S.K., Mohan, A. \& Kadhiravan, T. (2005) HIV-TB co-infection: Epidemiology, diagnosis and treatment. Indian Journal of Medical Research 121, 550-567.

Somi, G., Matee, M., Makene, C.L., van den Hombergh, J., Kilama, B., Yahya-Malima, K.I., Masako, P., Sando, D., Ndayongeje, J., Rabiel, B. \& Swai, R.O. (2009) Three years of HIV/AIDS care and treatment services in Tanzania: achievements and challenges. Tanzania Journal of Health Research 11, 136-143.

van den Broek, J., Mfinanga, S., Moshiro, C., O’Brien, R., Mugomela, A. \& Lefi, M. (1998) Impact of human immunodeficiency virus infection on the outcome of treatment and survival of tuberculosis patients in Mwanza, Tanzania. International Journal of Tuberculosis Lung Disease 2, 547-552

Williams, G.B. \& Dye, C. (2003) Antiretroviral therapy for TB control in the era of HIV/AIDS. Science 301, 1535-1537.

WHO (2008) Global Tuberculosis Control: Surveillance, Planning, Financing. WHO Report 2008. WHO/HTM/ TB/2008.393. World Health Organization, Geneva, 1-168.

WHO (2009) Rapid Advice: Antiretroviral Therapy for HIV Infection in Adults and Adolescents. WHO Report 2009. World Health Organization, Geneva, 1-25. 